\title{
鲜花香皇的制备
}

一推荐一个大学化学综合教学实验

马贤波, 李新汉, 张富琴, 陈名红 ${ }^{*}$, 叶艳青, 黄超

云南民族大学化学与环境学院, 昆明 650500

摘要：介绍了一个综合化学实验, 涉及丰产天然产物有效成分的提取、皇化反应、添加摚拌制备日化产品。该实验 将大学有机化学的基础知识、基本实验技能与日常生活相结合, 制备出鲜花香皇, 具有趣味性、系统性和连贯性。 大大激发了学生学习的兴趣, 加深了学生对有机化学基础知识的理解, 培养和提高了学生的创新能力和综合实践能 力。

关键词：天然产物有效成分；提取；㿝化反应；鲜花香皇；综合化学实验 中图分类号: G64; O6

\section{Preparation of Flower Soaps: A Recommendation of a Comprehensive Chemistry Experiment}

\author{
Xianbo Ma, Xinhan Li, Fuqin Zhang, Minghong Chen *, Yanqin Ye, Chao Huang * \\ School of Chemistry and Environment, Yunnan Minzu University, Kunming 650500, China.
}

\begin{abstract}
A comprehensive chemistry experiment was introduced, which involved the extraction of active ingredients of natural products and the preparation of daily chemical products by saponification reaction. This experiment systematically and coherently combined the basic knowledge of university organic chemistry, basic laboratory skills and life to prepare flower soaps. Through the experiment, students' interests and understanding of the knowledge of organic chemistry will be stimulated. Meanwhile, their ability of innovation will be improved.
\end{abstract}

Key Words: Effective components of natural products; Extraction; Saponification; Flower soaps;

Comprehensive chemistry experiment

近年来, 开发和利用天然产物有效成分, 绿色安全制造有利于人体健康的化学产品已成为工业 生产和化学教育的重要方向 ${ }^{[1]}$ 。鲜花作为简单易得的经济作物, 其含有的精油、色素等成分常添加 应用于日用化学品的制备中而广受大众青崃。例如知名度较高的多年生亚灌木, 具有 “芳香之王” 美誉的唇形科薰衣草属植物一一薰衣草, 现代研究表明薰衣草及其精油中的芳香成分具有抗菌、抗 焦虑、抗氧化、抗突变等功效 ${ }^{[2]}$, 为我们认识神奇化学世界和加工化学产品提供了天然材料。大多数 高校有机化学实验教材中包含 “菠菜色素的提取” “黄连素的提取” 等天然产物的提取实验, 涵盖 分离、提取、纯化等过程, 但是, 将提取天然产物应用于制备鲜花香㿝的教学实验却鲜有报道。因

收稿: 2020-09-07; 录用: 2020-11-03; 网络发表: 2020-11-26

“通讯作者, Emails: cmhkm99@163.com (陈名红); huangchao@ynu.edu.cn (黄超)

基金资助: 国家自然科学基金(21662046); 国家级大学生创新创业训练计划项目(s201910691023); 云南省大学生创新创业训练计划项目 (s201910691049) 
此, 我们结合实验可操作性和本校多年教学基础 ${ }^{[3,4]}$, 设计将提取到的鲜花色素和精油加入到透明皇 的制备当中，制成了日用的鲜花香皇产品。

本文介绍了鲜花香皇的制备, 该实验是集创新性、系统性、连贯性于一体的综合性实验, 很大 程度上提高了学生独立思考、动手操作和解决实际问题的能力。学生通过动手制作鲜花香皇, 将化 学知识充分运用于我们的日常生活中, 激发了学生的学习兴趣, 师生之间乐教乐学。

\section{1 实验目的}

(1) 通过薰衣草中色素、精油的提取和透明皇的制备三个实验了解天然产物中有效成分提取以 及分离提纯方法, 与此同时，掌握皇化反应基本原理;

(2) 掌握天然产物中有效成分提取、添加混合和透明皇制备的操作方法;

(3) 掌握溶剂浸提法、水蒸气蒸馏的基本原理和方法。

\section{2 实验原理}

吴霞课题组 ${ }^{[5]}$ 对薰衣草化学成分进行了研究, 实验将分离得到的黄酮类化合物在薄层色谱上进行 酸水解检识出葡萄糖, 证实为苷类。黄酮类化合物广泛分布于植物界中, 且易溶于水, 水溶液色泽鲜 艳且着色能力强 ${ }^{6]}$ 。基于此类化合物的物理性质, 可采用溶剂浸提法提取薰衣草色素。另外从薰衣草 中提取出来的薰衣草精油作为天然植物提取物, 包括芳樟醇、乙酸芳樟酯、乙酸薰衣草酯、薰衣草醇 和樟脑等主要成分 ${ }^{[7]}$ 。对于化学性质稳定的薰衣草精油则多采用水蒸气蒸馏法提取, 利用高温水蒸气 将精油从薰衣草中蒸馏出来, 再经冷凝形成乳白色的油水混合物, 最后利用油水不互溶原理经分液分 离混合液体。油脂在酸或碱的催化下会发生水解, 利用油脂的此类性质, 在氢氧化钠介质下经热诱 导, 硬脂酸甘油酯发生皇化反应生成硬脂酸钠, 即为肥㿝的主要成分 ${ }^{[8]}$ 。其反应式见图1。

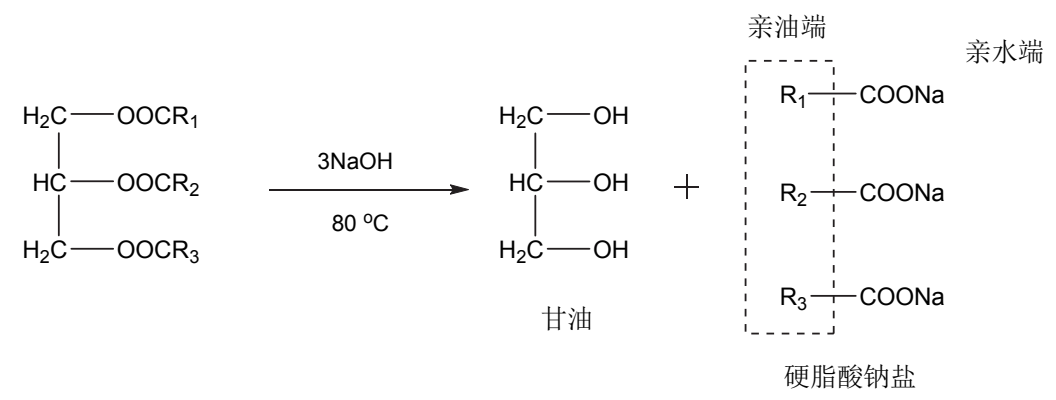

图1 硬脂酸皇化反应式

本实验的关键在于将三个实验进行创新性改进并串联为一个综合性实验, 以提高其效率、减少 试剂能耗和全过程提升。用树脂吸附提取可得到天然优质的薰衣草红色素 ${ }^{[9]}$, 但考虑到实际推广难 度, 不予采用此方法提取色素。查阅文献我们发现薰衣草色素和玫瑰花色素含有相同呈色功能的黄 酮类化合物, 因此借鉴了更利于操作的玫瑰花色素提取方法来提取薰衣草色素。结合相关文献报 道, 王方课题组 ${ }^{[10]}$ 通过单因素和正交试验研究表明：玫瑰花红色素提取的最佳条件为： $70 \%(\mathrm{~V} / \mathrm{V})$, 乙醇为溶剂, $\mathrm{pH}$ 值为 1.0 , 物料比 $(m / V)$ 为 $1: 8$, 浸提温度 $60^{\circ} \mathrm{C}$, 时间为 $1 \mathrm{~h}$ 。结合王方课题组的工作, 将提取到的天然产物有效成分加入到透明皇的制备当中, 通过实验发现在提取玫瑰花红色素的实验 中, 调节 $\mathrm{pH}$ 值为 3.0 时也有一样的效果。降低提取时酸的用量, 为后续㿝化反应降低碱的用量和实现 连贯综合制备鲜花香皇打下基础。

\section{3 仪器和试剂}

仪器：分析天平(上海天平仪器厂)、电炉(北京市永光明医疗有限公司); 旋转蒸发仪(N-1100D- 
$\mathrm{WD}$, 东京理化(日本))、烧杯 $(150 \mathrm{~mL} 、 50 \mathrm{~mL})$ 、量筒、玻璃棒、玻璃管、圆底烧瓶、三颈烧瓶、分 液漏斗、水蒸气发生装置、温度计、冷凝管、蒸馏头、尾接管、胶管若干、雉形瓶、模具(市售商品) 等，除特殊标注外均从上海仁科生物科技有限公司购买。

材料：鲜花(以薰衣草为例，市售的干薰衣草或者鲜薰衣草均可)、牛油(市售商品)。

试剂: 无水乙醇、蒸馏水 (课题组制备)、冰醋酸、菎麻油、氢氧化钠、蔗糖、丙三醇、 $\mathrm{NaCl}$ 、无 水 $\mathrm{Na}_{2} \mathrm{SO}_{4}$ 等。除特殊标注外, 试剂均为分析纯, 均购自阿拉丁生化科技股份有限公司。

其他: $\mathrm{pH}$ 试纸、称量纸、药匙、剪刀、镊子等, 均购自上海仁科生物科技有限公司。

\section{4 实验操作步骤}

\section{1 鲜花色素提取实验}

称取 $20.0 \mathrm{~g}$ 薰衣草, 洗净后剪碎放入雉形瓶中, 加入无水乙醇 $50 \mathrm{~mL}$ 并用冰醋酸调 $\mathrm{pH}$ 至 3.0 左右, 装入圆底烧瓶中, 在 $60{ }^{\circ} \mathrm{C}$ 下持续加热 $1 \mathrm{~h}$, 用纱布过滤以后得到薰衣草色素提取液, 重复2-3次反复 提取至无色，将所有提取液合并，旋蒸后得到薰衣草色素浓缩液。全程大致需要 $80 \mathrm{~min}$ 。

\section{2 鲜花精油提取实验}

称取干薰衣草 $200.0 \mathrm{~g}$ 按照花液比 $(m / V)$ 为 $1: 7$ 进行粉碎(其中 $m$ 单位为 $\mathrm{g}, V$ 单位为 $\mathrm{mL}$ ), 添加一定 量的 $\mathrm{NaCl}$ 溶液使之与粉碎液混合, 然后将其转移到蒸馏釜中进行薰衣草精油的提取。先回流 $1 \mathrm{~h}$, 然 后进行过滤。搭建水蒸气蒸馏装置, 将滤液进行水蒸气蒸馏 $30 \mathrm{~min}$, 得到油水混合物。对分层的馏 出液进行萃取, 在萃取液中加入少量无水 $\mathrm{Na}_{2} \mathrm{SO}_{4}$, 最后用旋转蒸发仪进行浓缩, 得到薰衣草精油, 全程大致需要 $120 \mathrm{~min}$ 。

\section{3 透明皇制作实验}

称取 $7.0 \mathrm{~g}$ 牛油, 加入 $12 \mathrm{~mL}$ 蒈麻油搅拌混合均匀, 再加入 $30 \%$ (质量百分比, 后文同) $\mathrm{NaOH} 9 \mathrm{~mL}$ 和 $95 \%$ 乙醇 $5 \mathrm{~mL}$, 水浴加热至 $80{ }^{\circ} \mathrm{C}$ 左右匀速搅拌 $10-15 \mathrm{~min}$, 待皇化反应完全后停止加热, 再加入 $2.0 \mathrm{~g}$ 甘油、 $3 \%$ 蔗糖 $8 \mathrm{~mL}$ 和 $5 \mathrm{~mL}$ 水, 不断搅拌混匀后降至 $60-70{ }^{\circ} \mathrm{C}$, 倒入冷模中冷却定型即可得到透 明㿝。全程大致需 $30 \mathrm{~min}$ 。

\section{4 鲜花香皇制作实验}

称取 $7.0 \mathrm{~g}$ 牛油, 加入 $12 \mathrm{~mL}$ 葛麻油搅拌混合均匀, 再加入 $20 \% \mathrm{NaOH} 9 \mathrm{~mL}$ 和 $60 \%$ 乙醇 $5 \mathrm{~mL}$, 水 浴加热至 $80^{\circ} \mathrm{C}$ 左右匀速搅拌 $10-15 \mathrm{~min}$, 待皇化反应完全后停止加热, 再加入 $2.0 \mathrm{~g}$ 甘油、3\%蔗糖 $8 \mathrm{~mL}$ 、 薰衣草色素浓缩液 $5 \mathrm{~mL}$ 、薰衣草精油浓缩液 5 滴, 不断搅拌混匀后降至 $60-70^{\circ} \mathrm{C}$, 倒入冷模中冷却定 型即可得到鲜花香㿝。

\section{5 结果与讨论}

\section{1 实验结果讨论}

由表1可知, 制备透明㿝和鲜花香皇所需时间以及加热温度差不多。在实验过程中, 制备鲜花香 㿝所需的 $\mathrm{NaOH}$ 溶液浓度和乙醇溶液浓度相比透明㿝的制备要稍低。从薰衣草中提取色素和精油的 实验, 反应温度和反应时间都是固定的, 危险性低, 重现性高, 便于教学推广。

表1 实验相关条件对比

\begin{tabular}{ccccc}
\hline 实验 & $\mathrm{NaOH} / \%$ & 乙醇 $/ \%$ & 温度 $/{ }^{\circ} \mathrm{C}$ & 时间 $/ \mathrm{min}$ \\
\hline 色素提取 & - & 70 & 60 & 80 \\
精油提取 & - & - & $>100$ & 120 \\
透明㿝的制备 & 30 & 95 & $60-80$ & 30 \\
鲜花香㿝的制备 & 20 & 60 & $60-80$ & 30 \\
\hline
\end{tabular}




\section{2 实际教学结果分析}

本科有机化学实验中如 “菠菜色素的提取” “黄连素的提取” “水蒸气的蒸馏” 等实验教学内 容以及考查范围有一定局限性, 无法达到综合性的教学目标, 并且实验过程枯燥乏味, 学生无法对 学习和实验产生兴趣, 教学和学习效果不佳, 因此我们设计了该综合性实验, 在串联三个已有实验 的同时, 进行相应改进达到相同的效果。本实验纳入本科教学实验后, 学生从鲜花色素的提取、鲜 花精油的提取、透明㿝的制备做起, 具有连贯性和系统性。学生参与整个实验过程, 制作出鲜花香 皇, 亲身体会到化学与我们的生活息息相关。实验取得了很好的教学效果, 不仅提升了学生的实验 技能和科研思维, 更提高了学生的创新能力和绿色环保意识。

\section{6 实验开设建议}

(1) 本实验面向化学类专业本科高年级学生开设, 可作为有机化学实验课程的选修实验教学 内容。由于本实验综合性很强, 完整做一次实验时间约为 $4 \mathrm{~h}$ 。一次实验课可有 $15-25$ 人参加, 每组 2-3人, 一年中开设两轮: 上半年4-6月, 下半年9-10月。实验集中在一天完成。本实验经验证, 大 概需要 5 个课时, 其中 1 个课时作为由教师做知识补充, 剩下 4 个课时完成实验部分。具体课时可分配 如下: 鲜花色素的提取 1 个课时, 鲜花精油提取 2 个课时, 最后制备鲜花香皇 1 个课时。

(2) 实验开始前一周, 指导教师布置学生查阅相关资料, 如天然产物的提取、水蒸气蒸馏、透明 㿝的制备等操作, 并完成思考题。实验进行时, 指导教师需提醒学生注意: 在制备鲜花香皇时, 要 控制好实验的温度; 在加入葛麻油时, 反应时间不能过长, 在加入 $20 \% \mathrm{NaOH}$ 和 $60 \%$ 乙醇后要迅速摚 拌, 并且要确保酯化反应完全时才可以停止加热。实验结束后要求学生完成实验报告。

(3) 通过本实验学生亲身经历鲜花香皇的制作过程, 从一开始的色素和精油的提取, 到完成一 块鲜花香㿝的制备, 使化学实验变得生动有趣, 大大激发了学生的积极性和学习热情。这样的综合 性实验, 锻炼了学生的综合能力、实践能力, 提高了学生的创新能力和实验技能。

\section{7 思考题}

(1) 提取薰衣草色素时为什么要调 $\mathrm{pH}$ 至3.0左右? 过高或过低会有什么影响?

(2) 提取薰衣草精油时为什么要先进行冷凝回流? 对产率有什么影响?

(3) 水蒸气蒸馏时温度如何控制? 没有蒸气进入三颈烧瓶中时要如何处理?

(4) 制备透明㿝中加入甘油、蔗糖的目的是什么?

(5) 制备透明皇时如何检验㿝化反应发生完全?

（6）制备透明㿝时温度过高或过低对皇化反应有何影响? 出现油脂不凝固应该如何处理? 油脂 凝固至无法搅动该如何处理?

\section{8 结语}

本实验结合了丰产天然产物鲜花色素和精油的提取以及透明皇的制备, 基于以上三个实验, 我 们设计了综合教学实验——鲜花香皇的制备, 该实验利用毒性低且便于获取的化学试剂代替性质相 似但有危害的试剂。通过该综合实验, 学生实现了绿色、高效、简便提取天然产物有效成分和制作 鲜花香皇全过程操作, 并对香皇日化产品有了更深入的认知。我们期望通过该综合实验, 达到提高 学生实验技能、拓展学生综合素质、寓教于乐的目的。

\section{参 考 文 献}

[1] 陈伟, 宣景宏, 孟宪军. 北方园艺, 2006, No. 2, 54.

[2] 李紫薇, 李芳, 粟有志, 管永正, 罗新泽, 韩凯乐, 张学超. 分析科学学报, 2020, 36 (3), 395. 
[3] 黄超, 杨丽娟, 蒋琳, 钏永明, 袁明龙. 有机化学实验. 北京: 科学出版社, 2016: 35 .

[4] 叶艳青, 郭俊明. 基础化学实验I. 杭州: 浙江大学出版社, 2014: 11-152.

[5] 吴霞, 刘净, 于志斌, 叶蕴华, 周亚伟. 化学学报, 2007, 65 (16), 49.

[6] 陈雅妮, 李琼, 任顺成, 郑舒文. 食品研究与开发, 2019, 40 (16), 63.

[7] 张丽, 孙越, 薄福民, 杨山景, 封安杰, 李凌军. 山东科学, 2020, 33 (5), 27.

[8] 陈佳. 化学教与学, 2014, No. 9, 96.

[9] 高燕, 袁琳. 昆明学院学报, 2012, 34 (6), 76.

[10] 王方, 吕镇城, 彭永宏. 华南师范大学学报, 2007, No. 4, 102. 